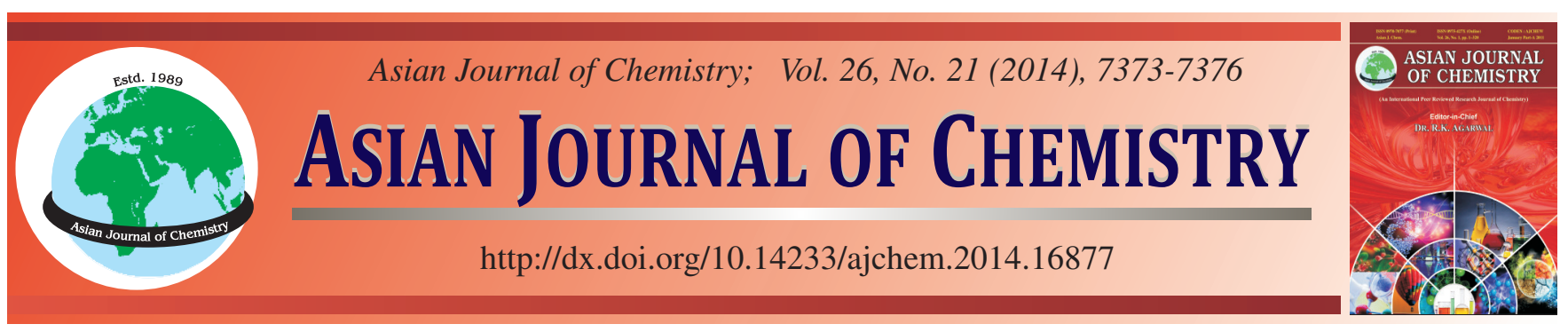

\title{
Preparation and Antibacterial Activity of Silver Nanoparticles Coated by Poly(vinyl pyrrolidone)
}

\author{
Guowen HE ${ }^{1,2}$, Qiufan Luo ${ }^{2}$, MengXiang Zhu² and Fenfang Li ${ }^{1, *}$
}

${ }^{1}$ School of Chemistry and Chemical Engineering, Central South University, Changsha 410083, P.R. China

${ }^{2}$ College of Chemical and Environmental Engineering, Hunan City University, Yiyang 413000, P.R. China

*Corresponding author: Fax: +86 731 88879616; Tel: +86 731 88836961; E-mail: zhongyihgw@163.com

Received: 16 December 2013;

Accepted: 3 April 2014;

Published online: 30 September 2014;

AJC-16140

\begin{abstract}
A silver nanoparticle with good monodispersity was produced by a convenient method for the reduction of silver nitrate in the presence of poly(vinyl pyrrolidone) as the surface modification agent. Silver nanoparticle had little effect on the chemical structure of poly(vinyl pyrrolidone). Silver nanoparticle modified by poly(vinyl pyrrolidone) with a nanosize of 5-10 nm had a face-centered cubic (FCC) crystalline structure and silver nanoparticle could develop a spherical morphology. Pure poly(vinyl pyrrolidone) have little antibacterial effect on bacteria and silver nanoparticle had perfect antibacterial activity with a wide antimicrobial spectrum against Gram-positive bacteria of Staphyloccocus aureus and Gram-negative bacteria of Escherichia coli. The antibacterial activity could be enhanced as increase of the silver nanoparticles concentration no matter what on Staphyloccocus aureus or Escherichia coli. Antibacterial activity of silver nanoparticles on Staphylococcus aureus was better than on Escherichia coli.
\end{abstract}

Keywords: Silver nanoparticles, Poly(vinyl pyrrolidone), Antibacterial activity, Preparation.

\section{INTRODUCTION}

Powder of silver and their polymer-based composites have been received considerable attention because of their excellent properties and broad applications ${ }^{1-3}$. Metallic silver nanoparticles (AgNP) attracted more and more attention due to the unique antibacterial properties and be widely used in medical and health field ${ }^{4}$. In the past two decades, many methods have been employed to prepare silver nanoparticle, such as citric acid reduction $^{5}$, electrochemical synthesis ${ }^{6}$, photochemistry ${ }^{7}$, ultrasound-assisted $^{8}$. In addition, poly(vinyl pyrrolidone) (PVP) as a stabilizer, organic solvents, such as ethanol, poly(ethylene glycol) ${ }^{4}$, N,N-dimethyl- formamide (DMF) ${ }^{9}$, citric $\operatorname{acid}^{10}$, had been used to prepare silver nanoparticle. Complex in the preparation process of silver nanoparticle produced between poly(vinyl pyrrolidone) and silver atoms. A protective layer of poly(vinyl pyrrolidone) on the surface of silver nanoparticle played a role to control the particle size, in which the poly(vinyl pyrrolidone) steric effect limits the reunion between the particles ${ }^{11}$. da Costa et al. ${ }^{12}$ reported that ultrafine silver nanoparticle with highly dispersion could be synthesized spontaneously in N,N-dimethyl acetamide (DMAc). Using N,N-dimethyl acetamide to prepare silver nanoparticle with high dispersion should be a very effective way.

In this paper, poly(vinyl pyrrolidone) had been taken as a stabilizer to prepare silver nanoparticle at high temperatures.
The chemistry structure, phase and morphology of resulting silver nanoparticle were characterized. In addition, in order to explore the antibacterial effects of silver nanoparticle powders, measurement of the zone of inhibition was employed.

\section{EXPERIMENTAL}

Preparation of silver nanoparticle coated by poly(vinyl pyrrolidone): In a typical experiment, $0.7500 \mathrm{~g}$ poly(vinyl pyrrolidone) and $0.2550 \mathrm{~g}$ silver nitrate were placed in a $300 \mathrm{~mL}$ conical flask and completely dissolved by joining $30 \mathrm{~mL}$ N,Ndimethyl acetamide with a stirring to $160{ }^{\circ} \mathrm{C}$ in the absence of light for $25 \mathrm{~min}$. The typical synthesis went through a number of colour changes before the colour became stable. The colour of the solution changed from light yellow to golden brown, to reddish brown and finally to dark red-brown. The reaction mechanism is as shown in Scheme-I.

The X-ray diffraction measurements were performed by using a Rigaku 2500 diffractometer with a wavelength of 1.54 A. Morphology of materials was imaged on a transmission electron microscope (TEM) of Cambridge S260. The FT-IR spectra were collected using a Nicolet 6700 and the wavenumber range was set from 4000 to $400 \mathrm{~cm}^{-1}$. The antimicrobial properties of the silver nanoparticle were assessed by zone of inhibition testing method using Gram-positive bacteria of Staphyloccocus aureus and Gram-negative bacteria of Escherichia coli. 


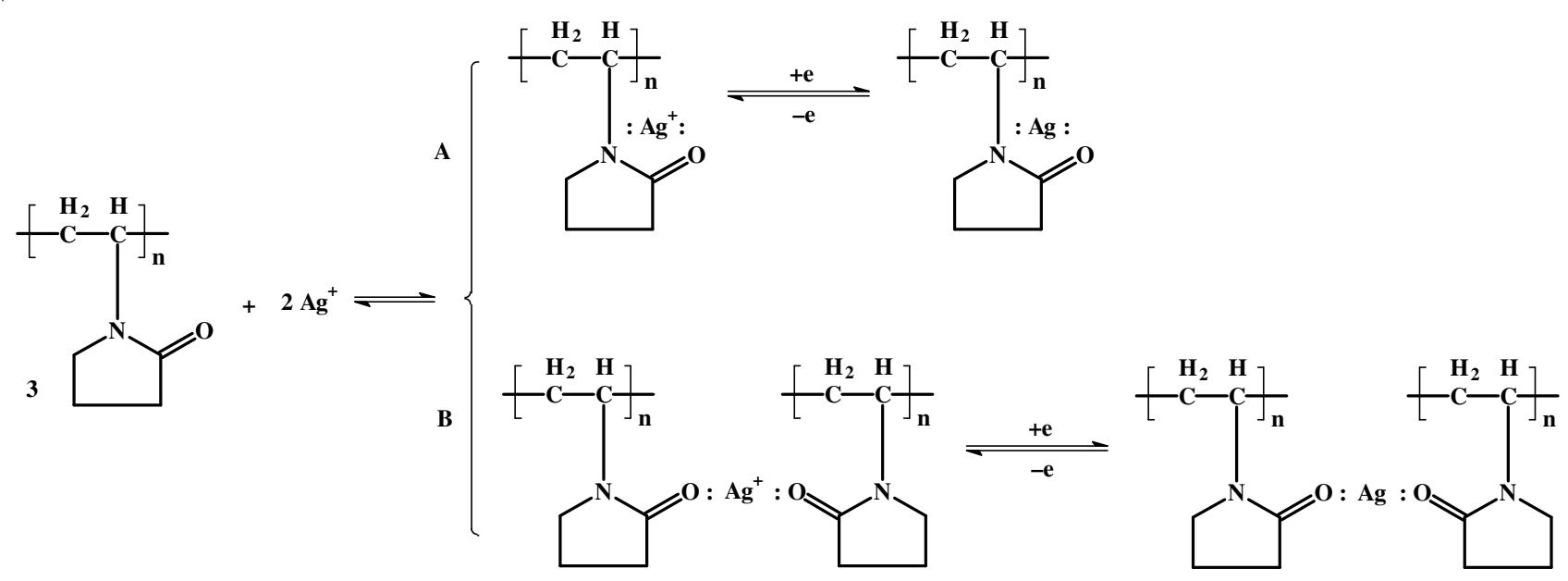

Scheme-I: Bonding mechanism of silver particles and poly(vinyl pyrrolidone)

\section{RESULTS AND DISCUSSION}

Chemical structure of silver nanoparticle coated by poly(vinyl pyrrolidone) and pure poly(vinyl pyrrolidone): The chemical structure of poly(vinyl pyrrolidone) and poly(vinyl pyrrolidone) containing silver particles had been investigated and their FTIR spectra are shown in Fig. 1. The characteristic peaks of methylene (-CH) stretching were clearly visible at 2954 and $2925 \mathrm{~cm}^{-1}$, indicating that the poly(vinyl pyrrolidone) alkyl chains have been adsorbed on the silver nanoplate surface. Vibration absorption peaks of the poly(vinyl pyrrolidone) belonged to chemical bonds of different groups. The strong vibration peak at $1652 \mathrm{~cm}^{-1}$ was attributed to the carbonyl group $(\mathrm{C}=\mathrm{O})$ stretching vibration. The absorption peak at 1289 $\mathrm{cm}^{-1}$ corresponded to the $\mathrm{C}-\mathrm{N}$ bond vibration. In addition, the absorption peak at $649 \mathrm{~cm}^{-1}$ indicates the existence of the swing to the face of the $\mathrm{C}-\mathrm{H}$ bond vibration peak ${ }^{11}$. Evidently, the broad peak at $3439 \mathrm{~cm}^{-1}$ can be assigned to the hydroxyl group $(-\mathrm{OH})$ stretching vibration of the solvent. The absorption peak positions and the transmittances Ag/PVP were almost the same as that of pure poly(vinyl pyrrolidone). This phenomenon indicates that the addition of silver nanoparticle into the poly(vinyl pyrrolidone) matrix has little effect upon the chemical structure of the poly(vinyl pyrrolidone).

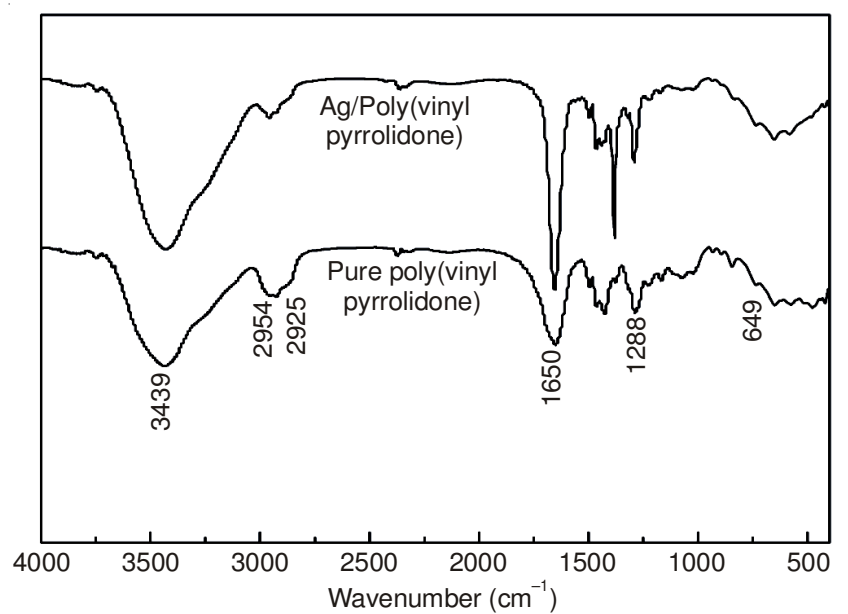

Fig. 1. FTIR spectra of poly(vinyl pyrrolidone) and silver nanoparticle coated by poly(vinyl pyrrolidone)
Phase and morphology of silver nanoparticle: Fig. 2 shows the XRD pattern of silver nanoparticle coated by poly(vinyl pyrrolidone). The diffraction peak of $2 \theta=23^{\circ}$ illustrated the existing of amorphous poly(vinyl pyrrolidone). Diagram for dispersion diffraction peaks around $2 \theta=38^{\circ}$, $44^{\circ}, 64.3^{\circ}$ and $77.3^{\circ}$, agreed well with the corresponding facecentered cubic (fcc) silver phase, i.e., (111), (200) (220) and (311) plane, respectively, indicating that the samples prepared by elemental silver successfully and the crystallization of silver nanoparticle completed relatively. On the contrary, the XRD patterns had not been appeared in the diffraction peaks of the impurity samples. It can attribute to the higher purity of the prepared samples.

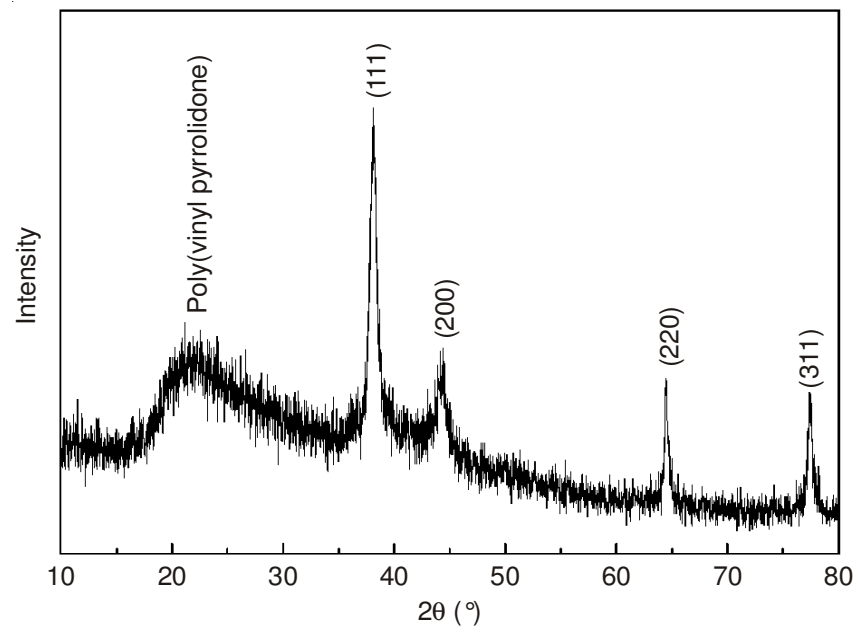

Fig. 2. XRD pattern of silver nanoparticle coated by poly(vinyl pyrrolidone)

The morphology of the resulting silver nanoparticle was observed by TEM techniques as showed in Fig. 3(a). The shape of all the synthesized silver particles irrespective of the size was spherical in nature. The particles had a favorable dispersion and no obvious aggregation. Moreover, the silver particles with a small size of 5-10 $\mathrm{nm}$ had relatively uniform size and narrow size distribution. Large grain in silver nanoparticle size and growth process went through the consumption of small particles corresponding to Ostwald ripening process ${ }^{13}$. With the introduction of poly(vinyl pyrrolidone), the particles of silver 
ions or atoms can coordinate with $\mathrm{N}$ or $\mathrm{O}$ in poly(vinyl pyrrolidone) and a covered layer of poly(vinyl pyrrolidone) can generate on the surface of the particles. Zhang et al. ${ }^{14}$ analyzed the possible mode of poly(vinyl pyrrolidone) protecting the particles and made an assumption that the two steps of $\mathrm{A}$ and B were equal (Scheme-I). With the increase of the silver nanoparticle diameter, the steric effect is strengthened. Both resonance peaks of $\mathrm{C}-\mathrm{N}$ and $\mathrm{C}=\mathrm{O}$ are changed obviously. With continuous generation of silver nanoparticle, silver ion is also continuously consumed, in which the electrons in the $\mathrm{O}$ atoms of poly(vinyl pyrrolidone) toward the $\mathrm{Ag}^{+} s p$ orbital in the formation of complexes ${ }^{14}$, indicating that not $\mathrm{N}$ but $\mathrm{O}$ atom of the poly(vinyl pyrrolidone) is coordinated with silver nanoparticle $^{15}$ and the reaction between silver nanoparticle and poly(vinyl pyrrolidone) only agreed with step B. The $s p$ orbit of $\mathrm{Ag}$ atom to form the coordination role with $\mathrm{O}$ atoms of poly(vinyl pyrrolidone) was weaker than that of $\mathrm{Ag}^{+}$ions. The coordination between the $\mathrm{N}$ atom of poly(vinyl pyrrolidone) and the empty orbital of the silver atoms would be generated ${ }^{15}$. When the particles grow up, the steric effect was important in the reaction of silver and poly(vinyl pyrrolidone). As a result, coordination between silver and $\mathrm{O}$ atoms is in poly(vinyl pyrrolidone) instead of $\mathrm{N}$.

In addition, the electron diffraction pattern, as showed in Fig. 3(b), clearly showed the crystalline nature of the particles. Accordingly, an average lattice parameter of $0.4073 \mathrm{~nm}$ for the FCC Ag and average atomic size of $0.1439 \mathrm{~nm}$ was estimated.
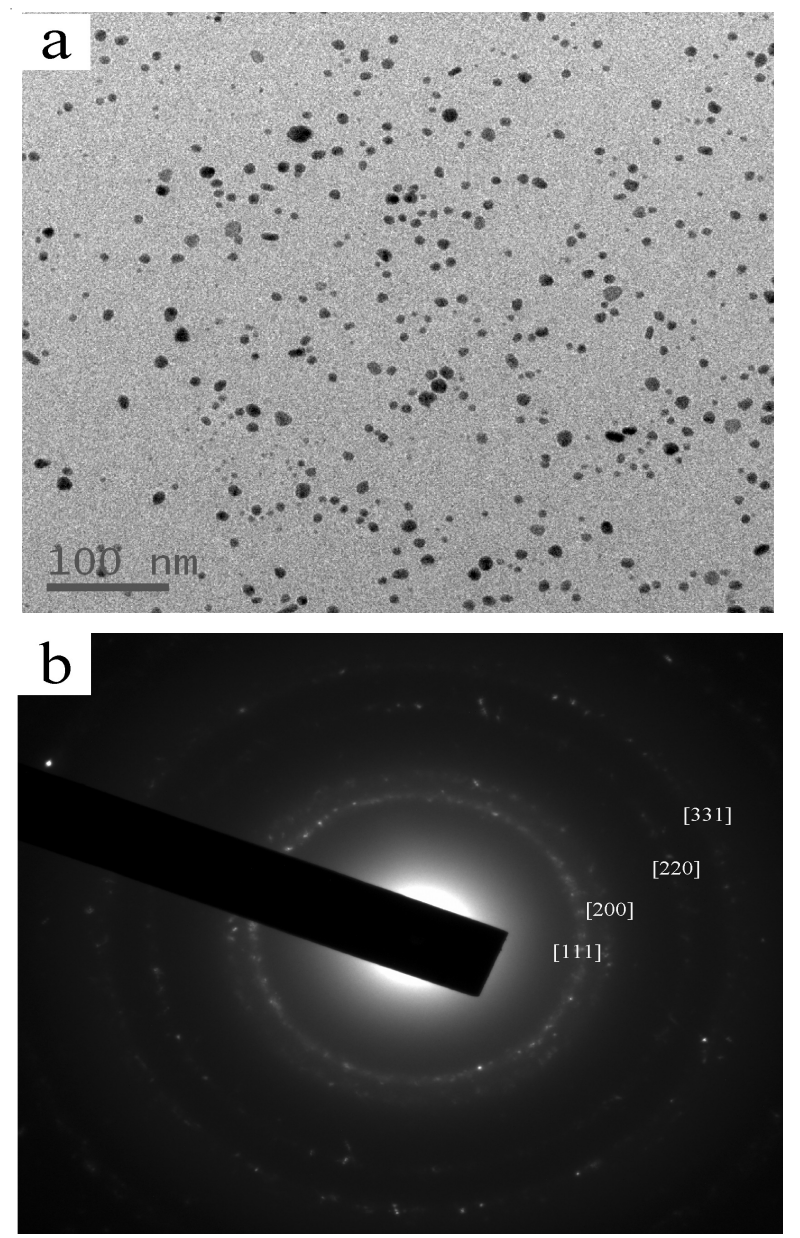

Fig. 3. TEM micrograph (a) and electron diffraction patterns of $\operatorname{AgNP}(b)$
Antibacterial activity of silver nanoparticle: Antibacterial activity of silver nanoparticle was studied against Staphylococcus aureus and Eschericia coli at different concentration. The concentration of bacteria was $10^{8} \mathrm{cfu} / \mathrm{mL}$. The solution of Ag/poly(vinyl pyrrolidone) was centrifugated at $10000 \mathrm{rpm}$ for $20 \mathrm{~min}$. Afterwards, the isolate was dissolved with N,N-dimethyl acetamide. The bacteria were daubed on the top of the medium, then some small cyclo-filter paper put on it in proper order. The silver nanoparticle were homogenously dispersed and stable immobilized on filter paper. Prepared mediums were reversed and incubated at $37^{\circ} \mathrm{C}$ for $24 \mathrm{~h}$.

Fig. 4 shows that the concentration of the silver nanoparticle can affect the size of the bacteriostatic circle. Obviously, the pure poly(vinyl pyrrolidone) have little antibacterial effect on both bacteria and silver nanoparticle had perfect antibacterial activity against Gram-positive bacteria of Staphyloccocus aureus and Gram-negative bacteria of Escherichia coli, indicating a wide antimicrobial spectrum existed in silver nanoparticle. The greater the diameter of bacteriostatic ring of the medium colour and fresh medium colour enlarged with the increase of the silver nanoparticles concentration, which indicated that the antibacterial activity could be enhanced as increase of the silver nanoparticles concentration no matter what on Staphyloccocus aureus or Escherichia coli. Moreover, inhibiting effect of silver nanoparticles against two bacteria was different. It is easy to see that the diameter of bacteriostatic ring against Staphyloccocus aureus was larger than that of Escherichia coli with the same silver nanoparticles concentrations. The antibacterial activity of silver nanoparticles on Staphylococcus aureus was better than on Escherichia coli.

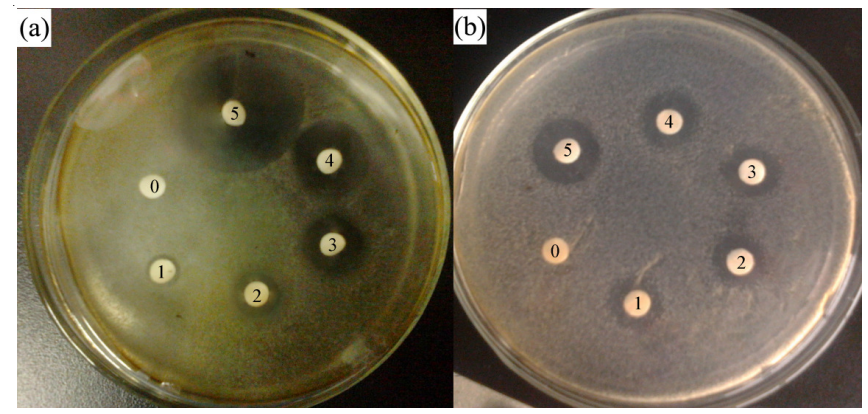

Fig. 4. Comparison of Staphyloccocus aureus (a) and Escherichia coli (b) inhibition zones formed by pure poly(vinyl pyrrolidone) (0) and different silver nanoparticle concentrations of $5 \mu \mathrm{g} / \mathrm{mL}(1), 25 \mu \mathrm{g} /$ $\mathrm{mL}$ (2), $50 \mu \mathrm{g} / \mathrm{mL}$ (3), $100 \mu \mathrm{g} / \mathrm{mL}$ (4) and $500 \mu \mathrm{g} / \mathrm{mL}$ (5)

\section{Conclusion}

A spherical silver nanoparticle with high dispersion and homogeneity had been prepared, in which poly(vinyl pyrrolidone) is used as a stabilizer. Silver nanoparticle had little effect on the chemical structure of poly(vinyl pyrrolidone). silver nanoparticle coated by poly(vinyl pyrrolidone) with a nanosize of 5-10 nm possessed a face-centered cubic (FCC) crystalline structure. Both of silver ion and silver atoms can coordinate with $\mathrm{N}$ and $\mathrm{O}$ atoms of poly(vinyl pyrrolidone) polar lactam group. The antibacterial activity of poly(vinyl pyrrolidone) and silver nanoparticle had been investigated. Silver nanoparticle had perfect antibacterial activity with a wide anti- 
microbial spectrum against Gram-positive bacteria of Staphyloccocus aureus and Gram-negative bacteria of Escherichia coli. The antibacterial activity could be enhanced as increase of the silver nanoparticles concentration no matter what on Staphyloccocus aureus or Escherichia coli. The antibacterial activity of silver nanoparticles on Staphylococcus aureus was better than on Escherichia coli. The nanocomposite of silver nanoparticle should be used importantly in the medical and health field.

\section{ACKNOWLEDGEMENTS}

The authors acknowledge the Postdoctoral fund of Central South University of China (No.126223) and the Natural Science Foundation of Hunan Province of China (NSF, Grant No.13JJ4102) for financial support.

\section{REFERENCES}

1. F.-C. Yang, K.-H. Wu, J.-W. Huang, D.-N. Horng, C.-F. Liang and M.-K Hu, Mater. Sci. Eng. C, 32, 1062 (2012).

2. B. Jiang, C. Tian, G. Song, W. Chang, G. Wang, Q. Wu and H. Fu, J. Mater. Sci., 48, 1980 (2013).
3. B. Pant, H.R. Pant, D.R. Pandeya, G. Panthi, K.T. Nam, S.T. Hong, C.S. Kim and H.Y. Kim, Colloids Surf. A, 395, 94 (2012).

4. L. Li, J. Sun, X. Li, Y. Zhang, Z. Wang, C. Wang, J. Dai and Q. Wang, Biomaterials, 33, 1714 (2012).

5. P.S. Mdluli, N.M. Sosibo, P.N. Mashazi, T. Nyokong, R.T. Tshikhudo, A. Skepu and E. van der Lingen, J. Mol. Struct., 1004, 131 (2011).

6. N.A. Kotov, M.E.D. Zaniquelli, F.C. Meldrum and J.H. Fendler, Langmuir, 9, 3710 (1993).

7. S. Saha, A. Pal, S. Kundu, S. Basu and T. Pal, Langmuir, 26, 2885 (2010).

8. J. Zhang, B. Han, M. Liu, D. Liu, Z. Dong, J. Liu, D. Li, J. Wang, B. Dong, H. Zhao and L. Rong, J. Phys. Chem. B, 107, 3679 (2003).

9. I. Pastoriza-Santos and L.M. Liz-Marzán, Nano Lett., 2, 903 (2002).

10. C. Munro, W. Smith, M. Garner, J. Clarkson and P. White, Langmuir, 11, 3712 (1995).

11. J. Bregado-Gutiérrez, A.J. Saldívar-García and H.F. López, J. Appl. Polym. Sci., 107, 45 (2008).

12. L.P. da Costa, A.L.B. Formiga, I.O. Mazali and F.A. Sigoli, Synth. Met., 161, 1517 (2011).

13. F. Bonet, K. Tekaia-Elhsissen and K.V. Sarathy, Bull. Mater. Sci., 23, 165 (2000).

14. Z. Zhang, B. Zhao and L. Hu, J. Solid State Chem., 121, 105 (1996).

15. H. Wang, X. Qiao, J. Chen, X. Wang and S. Ding, Mater. Chem. Phys., 94, 449 (2005). 